\title{
Morphine-induced locomotor sensitization produces structural plasticity in the mesocorticolimbic system dependent on CB1-R activity.
}

Thomas Guegan $^{1}$, Joan Pau Cebrià ${ }^{1}$, Rafael Maldonado ${ }^{1} \&$ Miquel Martin ${ }^{{ }^{*}}$.

1 Laboratory of Neuropharmacology, Parc de Recerca Biomèdica de Barcelona/Universitat Pompeu Fabra (PRBB/UPF), C/ Dr. Aiguader 88, 08003, Barcelona, Spain.

${ }^{*}$ Current address: Pathophysiology of synaptic transmisión laboratory, Institut de Génomique Fonctionnelle; 141, Rue de la Cardonille, 34094, Montpellier, France.

\section{Correspondence to:}

Rafael Maldonado, Laboratory of Neuropharmacology, Parc de Recerca Biomèdica de Barcelona/Universitat Pompeu Fabra (PRBB/UPF), C/ Dr. Aiguader, 88, 08003, Barcelona, Spain. Telf: +(34) 933160824; FAX: +(34) 933160901; E-mail address: rafael.maldonado@upf.edu;

Miquel Martin, Pathophysiology of synaptic transmission laboratory, Institut de Génomique Fonctionnelle; 141, Rue de la Cardonille, 34094, Montpellier, France. Telf: +(33) 0434359210; FAX: +(33) 0434359200; E-mail address: miquel.martin@igf.cnrs.fr 


\section{ABSTRACT}

Changes in structural plasticity produced by the chronic exposure to drugs of abuse, such as alterations in dendritic spine densities, participate in the development of maladaptive learning processes leading to drug addiction. Understanding the neurobiological mechanisms involved in these aberrant changes is crucial to clarify the neurobiological substrate of addiction. Druginduced locomotor sensitization has been widely accepted as a useful animal model to study these mechanisms related to drug addiction. We have evaluated the changes in structural plasticity in the mesocorticolimbic system involved in morphine-induced locomotor sensitization. The role of the cannabinoid receptor type-I (CB1-R) in these neuroplastic alterations has also been studied using CB1-R deficient mice (CB1-R KO). Structural plasticity changes promoted by morphine are a highly dynamic phenomenon that evolves during the entire time course of the behavioural sensitization in wild-type (WT) animals. The different phases of the sensitization process were related to specific changes in connectivity between neurons revealed by modifications in dendritic spines in specific areas of the mesocorticolimbic system. Moreover, the lack of morphine-induced locomotor sensitization in CB1-R KO mice was accompanied by abnormal alterations in structural plasticity in the same mesocorticolimbic areas. These specific structural plasticity changes mediated by CB1-R activity seem necessary for the normal progression of morphine-induced locomotor sensitization, and could play a critical role in the addictive process.

Keywords: Addiction, Cannabinoid type-I receptor, dendritic spines, locomotor sensitization, opioid, structural plasticity. 


\section{INTRODUCTION}

Drug addiction is characterized by the presence of maladaptive long-lasting behavioral alterations, including compulsive drug intake and recurrent drug seeking episodes, that can persist even for a lifetime period (Robinson and Berridge, 2003; Koob and Le Moal, 2001). These maladaptive responses are hypothesized to be due to aberrant learning processes in specific areas of the brain reward system as a consequence of the chronic exposure to the drug (Hyman and Malenka, 2001; Kalivas and O'Brien, 2008; Russo et al., 2010; Lüscher and Malenka, 2011). Neuroplasticity changes, mainly link to glutamatergic neurotransmission have been proposed to be key neurobiological mechanisms underlying these learning processes during drug addiction (Rampon and Tsien, 2000; Robinson and Kolb, 2004). Most glutamatergic inputs into neurons occur at the level of the dendritic spines (Harris and Kater, 1994). Enduring alterations in glutamatergic synaptic connectivity occur when the number and/or morphology of the dendritic spines are modified, what is also called structural plasticity changes (Leuner et al., 2003; Buxbaum et al., 2014; Enriquez-Barreto et al., 2014). Thus, modifications in synaptic efficacy as a consequence of specific changes in structural plasticity in neurons of the brain reward system are suggested to be crucial for aberrant learning processes during drug addiction (Russo et al., 2010). In agreement, chronic exposure to different drugs of abuse has been reported to modify structural plasticity in mesocorticolimbic neurons (Robinson and Kolb, 1997; Crombag et al., 2005; Russo et al., 2010). To clarify the mechanism that triggers the progress of these stable neuroplastic modifications represents a crucial topic for better understanding how chronic drug exposure leads to drug addiction. An useful approach to investigate these adaptive changes produced by drugs of abuse is the model of drug-induced behavioral sensitization in mice. Behavioral sensitization is usually revealed by the increased locomotor response when repeatedly exposed to a drug, and has been proposed to be underlined by the same neuronal mechanisms involved in drug seeking relapse (Vanderschuren and Pierce, 2010; Steketee and Kalivas, 2011). Opioids are among the drugs 
that produce locomotor sensitization (Vanderschuren and Kalivas, 2000). Locomotor sensitization has been reported to correlate with the morphological synaptic changes promoted in dendritic spines in different areas of the mesocorticolimbic system (Li et al., 2004; Robinson and Kolb, 2004).

The endogenous cannabinoid system is an important nerobiological system involved in controlling a wide range of biological processes, such as motor activity, cognition, emotional responses and neural plasticity. This system consists of at least 2 different cannabinoid receptors, known as $C B 1-R$ and $C B 2-R$, the endogenous ligands, known as endocannabinoids (mainly anandamide and 2-arachidonyl-glycerol) and the proteins and enzymes responsible for their synthesis and degradation (De Petrocellis and Di Marzo; 2009). Previous studies have described the involvement of the CB1-R in modulating the pharmacological effects of different drugs of abuse. Thus, CB1-R activation is crucial for the development of opioid-induced locomotor sensitization and CB1-R KO mice do not develop this morphine sensitization (Martin et al., 2000). Moreover, CB1-R are important modulators of structural plasticity changes in the mesocorticolimbic system (Guegan et al., 2013), and are also involved in neuroplasticity induced by drugs of abuse, such as cocaine (Ballesteros-Yañez et al., 2007).

In this study, we have investigated the specific changes in structural plasticity in different mesocorticolimbic areas occurring during morphine-induced locomotor sensitization and the involvement of CB1-R in these adaptive changes. For this purpose, we have evaluated structural plasticity in the mesocorticolimbic system in WT and CB1-R KO mice exposed to a protocol of morphine-induced locomotor sensitization. We observed that locomotor sensitization was associated to alterations in neuroplasticity in the mesocorticolimbic system that were dependent on CB1-R. These neuroplastic modifications after morphine exposure occur in WT, but not in CB1-R KO mice, and might play a crucial role in the development of 
behavioral sensitization, and subsequently, in the progress towards drug-induced seeking behavior and relapse. 


\section{MATERIAL AND METHODS}

\section{Animals}

The experiments were carried out in C57BL6/J background male CB1-R KO and WT littermates mice from 8 to 12 weeks old at the beginning of the experiments. The generation of CB1-R KO mice was described previously (Zimmer et al., 1999). Briefly, homozygous WT and CB1-R KO mice were bred by backcrossing of chimeric and heterozygous animals to C57BL6/J and interbreeding of heterozygous animals for at least 10 generations in order to obtain a pure C57BL6/J background. The animals were housed 4 per cage and maintained in a controlled temperature $\left(21 \pm 1^{\circ} \mathrm{C}\right)$ and humidity $(55 \pm 10 \%)$ room with a $12: 12$-h light/dark cycle (on at 8 a.m. and off at 8 p.m.). Animals had ad libitum access to food and water during the entire experimental procedure. Mice were habituated to the experimental room and handled for one week before starting the experiments. All animal procedures were conducted in accordance with the standard ethical guidelines (European Communities Directive 86/60-EEC, Animal Welfare Assurance \#A5388-01, IACUC Approval Date 06/08/2009) and approved by the local ethical committee (Comitè Ètic d'Experimentació Animal-Institut Municipal d'Assistència Sanitària-Universitat Pompeu Fabra). All the experiments were performed under blind conditions.

\section{Drugs}

Morphine was provided by the Ministerio de Sanidad y Consumo (Spain), dissolved in saline (0.9\%) and administered subcutaneously (s.c.) at the dose of $15 \mathrm{mg} / \mathrm{kg}$. 


\section{Locomotor sensitization procedure}

Morphine-induced locomotor sensitization was evaluated as previously reported (Martin et al., 2000). We used locomotor activity boxes $(9 \times 20 \times 11 \mathrm{~cm}$; Imetronic, France $)$ containing a line of photocells $2 \mathrm{~cm}$ above the floor to measure horizontal movements, and another line located $6 \mathrm{~cm}$ above the floor to measure vertical activity (rearing). Mice were individually placed in the boxes and the total activity (ambulatory movements plus small movements) was recorded during $15 \mathrm{~min}$ in a low luminosity environment (20-25 lux). All the locomotor activity tests were performed between 09:30 and 10:30 h.

Basal locomotor activity was measured on days 1, 2 and 3 in order to habituate the animals to the test environment and to obtain a stable baseline. On day 4, chronic treatment with morphine (15 mg/kg, s.c., twice daily) or vehicle (control mice) began for a period of 15 days. Morning injections were performed between 9.30 and $10.30 \mathrm{~h}$, and evening injections between 18.00 and $19.00 \mathrm{~h}$. The acute locomotor effects of morphine were evaluated on day 4, 10 min after injection. During chronic drug treatment, locomotion was recorded every 3 days (days 7, 10, 13, 16 and 19) 10 min after the morning injection. One week after chronic drug treatment (day 26), saline and morphine treated animals from both genotypes were divided in different experimental groups with similar locomotor activities: half of the mice were treated again with saline (SALINE/SALINE GROUP and MORPHINE/SALINE GROUP) and half received an acute morphine administration $(15 \mathrm{mg} / \mathrm{kg}$, s.c.) (SALINE/MORPHINE GROUP and MORPHINE/MORPHINE GROUP). Locomotor activity was measured again ten min after the injection.

\section{Ballistic labeling with the fluorescent dye Dil}

Immediately after locomotor evaluation on day 26 , mice were deeply anesthetized by an intraperitoneal (i.p.) injection $(0.2 \mathrm{ml} / 10 \mathrm{~g}$ body weight) of a mixture of ketamine $(100 \mathrm{mg} / \mathrm{kg})$ 
and xylazine $(20 \mathrm{mg} / \mathrm{kg})$ and rapidly perfused intracardiacally using a peristaltic pump delivering at $20 \mathrm{ml} / \mathrm{min}$. Ten $\mathrm{ml}$ of $\mathrm{Na} 2 \mathrm{HPO} 4 / \mathrm{NaH} 2 \mathrm{PO} 4 / \mathrm{NaCl}$ buffer (PBS) $0.1 \mathrm{~m}, \mathrm{pH} 7.5$ was initially perfused followed by $40 \mathrm{ml}$ of $4 \%$ paraformaldehyde (PFA) in PBS $0.1 \mathrm{M}, \mathrm{pH}$ 7.5. Brains were quickly removed from the skull and postfixed in $4 \%$ PFA for $10 \mathrm{~min}$. Brain coronal sections $(100 \mu \mathrm{m})$ containing the nucleus accumbens (NAC) (Paxinos and Franklin, 2001) (from bregma $1.54 \mathrm{~mm}$ to bregma $1.10 \mathrm{~mm}$ ) or the medial prefrontal cortex (mPFC) (from bregma $1.98 \mathrm{~mm}$ to bregma $1.70 \mathrm{~mm}$ ) were obtained by using a vibratome (Leica VT $1000 \mathrm{~S}$, Nussloch, Germany) and kept in PBS 0.1 M until processed for fluorescent labeling. Brain slices were labeled by ballistic delivery of fluorescent dye Dil (Molecular Probes, Eugene, OR, USA) using a gene gun apparatus (Helios Gene Gun System, Bio-Rad, Deutschland), as described previously (Grutzendel et al., 2003), and postfixed with PFA for 4 hours at room temperature to further preserve structures and to allow the diffusion of the dye Dil. Sections were placed on microscope gelatine-coated slides and coverslipped with mounting medium (Mowiol). Then, images were acquired with confocal microscope (Zeiss LSM 510, Germany) with an oil immersion lens (40x) to analyze dendritic spine density and structure.

\section{Dendritic spine analysis}

Individual medium spiny neurons (MSN) in the NAc and pyramidal neurons from the mPFC were chosen for spine analysis based on several criteria, as described previously (Lee et al., 2006). Briefly, individual MSN in the NAC and mPFC pyramidal neurons were chosen for spine analysis based on the following criteria (i) there was minimal or no overlap with other labeled cells to ensure that processes from different cells would not be confused, (ii) at least three primary dendrites needed to be visible for cells to be used for analysis and (iii) distal dendrites (from secondary dendrites to terminal dendrites) were examined. Dendrites from MSN in the core and shell of the NAc (from bregma 1.54 to bregma 1.10) and basilar dendrites of 
pyramidal neurons taken predominantly from the prelimbic and infralimbic areas of the MPFC (from bregma 1.98 to bregma 1.70) were analyzed.

To calculate spine density, a length of dendrite (at least $20 \mu \mathrm{m}$ long) was traced by using a confocal microscope (Zeiss LSM 510, Germany) with an oil immersion lens (40x). All images of dendrites were taken at different $z$ levels ( $0.3 \mu \mathrm{m}$ depth intervals) to examine the morphology of dendritic spines. All measurements were made using IMAGE J analysis software. Protrusions from dendrites were classified into five types based on their morphology: class 1 protrusions, also called stubby protuberances were $0.5 \mu \mathrm{m}$ in length, lacked a large spine head, and did not appear to have a neck; class 2, or mushroom-shaped spines were between 0.5 and $1.25 \mu \mathrm{m}$ in length and were characterized by a short neck and large spine head; class 3, or thin spines ranged between 1.25 and $3.0 \mu \mathrm{m}$ and had elongated spine necks with small heads; class 4 , or wide spine were between 0.5 and $1.25 \mu \mathrm{m}$ in length and were characterized by a large neck and a large spine head; and class 5 or branched spine ranged between 1.25 and $3.0 \mu \mathrm{m}$ and had elongated spine necks with two or more spine heads.

\section{Statistical analysis}

Behavioural data were analyzed by using three-way or two-way ANOVA with genotype (between subjects) and/or treatment (between subjects) and day (within subjects) as factors of variations (Table 2). Morphological data were analyzed by using a two-way ANOVA with genotype and treatment as factors of variation (between subjects) (Table 3). Post hoc comparisons were performed by using the Fischer F-test when appropriate. Statistical tests were performed with Statistica@ (StatSoft, Tulsa, OK, USA). 


\section{RESULTS}

Genetic deletion of CB1-R modifies basal locomotor activity and prevents the development of morphine locomotor sensitization.

Inactivation of CB1-R activities in mice produced hypolocomotor effects, in agreement with previous studies (Zimmer et al., 1999; Martin et al., 2000). Thus, total locomotion in CB1-R KO animals was lower than in WT mice on the 3 days of habituation (Fig. 1A; $F_{(1,117)}=779.17$; $\mathrm{p}<0.01$ ). Acute morphine administration increased locomotor activity in both genotypes (Fig. 1B; WT $F_{(1,30)}=65.083 ; p<0.001 ; C B 1-R$ KO $\left.F_{(1,27)}=70.783 ; p<0.001\right)$. However, acute morphineinduced hyperlocomotion was 5 times higher in CB1-R KO than in WT mice in proportion to their respective basal activity on day 3 (Fig. $1 B$ and $C ; F_{(1,58)}=33.439 ; p<0.001$ ). These data suggest that $\mathrm{CB1}-\mathrm{R}$ participate in morphine acute locomotor effects. In contrast, chronic morphine treatment leads to sensitization of its locomotor effects only in WT, but not in CB1-R KO animals that showed a significant locomotor decrease (Fig. 1B day 19; WT $F_{(1,30)}=15.030$; $p<0.01 ; C B 1-R$ KO $F_{(1,27)}=7.777 ; p<0.05$ (repeated measure), as previously reported (Martin et al., 2000).

One week after chronic morphine treatment, mice were challenged with the same dose of morphine (15 mg/kg, s.c.) to evaluate the maintenance of the behavioral sensitization (day 26 ). Sensitized locomotor responses to morphine were maintained for at least one week after the cessation of the chronic treatment in WT mice (Fig. 1B and C), and this response was significantly diminished in CB1-R KO mice (Fig. $1 B$ and $C ; F_{(1,34)}=32.924 ; p<0.001$ ). Acute morphine administration on day 26 in animals chronically treated with saline produced the same hyperlocomotor effects in both genotypes than on day 4 (Fig. 1B and C). 


\section{Genetic deletion of CB1-R modifies basal neuronal morphology in the mesocorticolimbic} system and exacerbates the changes induced by acute morphine.

Changes in dendritic spine densities in MSN from the NAc shell and core and pyramidal neurons from the mPFC, both areas involved in drug-induced behavioral sensitization, were analyzed after the locomotor test on day 26. Dendritic spine densities in WT and CB1-R KO control groups (SALINE/SALINE treated animals) revealed that deletion of CB1-R altered total spine densities in MSN of the NAc core, but not in NAc shell or mPFC (Fig. 2). Total spine density in the NAc core was decreased in CB1-R KO control mice when compared to WT mice (Fig. 2C and $\left.D ; F_{(1,95)}=4.816 ; p<0.005\right)$. A significant increase in stubby- and a decrease in mushroom-type of spines $\left(F_{(1,89)}=19.952 ; p<0.001\right.$ and $F_{(1,89)}=13.751 ; p<0.001$ respectively) were also observed in the NAC shell in CB1-R KO mice when compared with WT animals in basal conditions (Fig. 3).

Our morphological analysis revealed that acute morphine administration $(15 \mathrm{mg} / \mathrm{kg}$, s.c.) did not modify total dendritic spines densities in any of the three brain areas studied in WT mice. In contrast, the same morphine administration significantly increased total dendritic spines densities in the NAc shell $\left(F_{(1,87)}=4.141 ; p<0.05\right)$ and core $\left(F_{(1,93)}=4.452 ; p<0.05\right)$ of CB1-R KO mice (Fig. 2) revealing the modulatory role of CB1-R in acute morphine effects in structural plasticity in the NAc MSN. We then evaluated the particular types of spines that result involved in these morphine structural plasticity changes. Acute morphine specifically increased mushroom-type dendritic spines density in the NAc shell $\left(F_{(1,93)}=8.950 ; p<0.01\right)$ and stubbytypes dendritic spines in the NAc core $\left(F_{(1,93)}=9.016 ; p<0.01\right)$ in CB1-R KO mice (Fig. 3 and 4 and Table 1). Although global changes in dendritic spines density were not revealed in WT mice after single morphine injection, a detailed analysis showed that acute morphine increased the proportion of stubby-type spines in the NAc shell (Fig. 3; $F_{(1,91)}=10.067 ; p<0.01$ ) and mushroomtype (Fig. 4; $F_{(1,91)}=4.210 ; p<0.05$ ) in the NAc core in WT mice. Therefore, our results show that 
acute morphine exposure modifies structural plasticity in the mesocorticolimbic system by altering the morphology of pre-existing spines. In addition, acute morphine-induced structural changes in the mesocoticolimbic circuit are regulated by the activity of the endogenous cannabinoid system, and the lack of CB1-R activities exacerbates these neuronal adaptations (Fig. 2).

\section{The lack of CB1-R differentially alters structural plasticity changes induced by chronic morphine in the NAc and mPFC.}

Previous studies have described that a challenge injection of morphine one week after the cessation of the chronic treatment leads to an increase in the sensitized locomotor response to this drug in mice (Martin et al., 2000). Structural plasticity analysis revealed that one week withdrawal from morphine treatment did not modify the total density nor the percentage of each sub-type of dendritic spines in the NAc shell and core of WT mice (WT MORPHINE/SALINE group) when compared with control animals (SALINE/SALINE). Similarly, no major structural plasticity changes were found in the NAc core and shell after chronic morphine treatment and withdrawal in CB1-R KO mice (MORPHINE/SALINE), although an increased proportion of stubby-types spines was revealed in the NAc core in these animals (Fig. 2, Fig. 4 and Table 1; $\left.F_{(1,87)}=7.250 ; p<0.01\right)$.

In contrast, disruption of chronic morphine treatment (MORPHINE/SALINE group) was associated with a significant increase in the dendritic spine density in the MPFC in WT (Fig. 2; $\left.F_{(1,96)}=8.456 ; p<0.01\right)$ and CB1-R KO mice $\left(F_{(1,89)}=6.378 ; p<0.05\right)$ when compared with their respective control groups. These changes were mainly due to an increase in the density of thin-types spines in both genotypes (Fig. 5 and Table $1 ; F_{(1,96)}=13.212 ; p<0.001 ; F_{(1,89)}=10.786$; $\mathrm{p}<0.01$, respectively), and were significantly higher in WT than in CB1-R KO mice (Fig. 2; total 
density WT vs KO $\left.F_{(1,90)}=4.159 ; p<0.05\right)$, suggesting that $C B 1-R$ participate in the generation of these plasticity changes.

Finally, a challenge morphine injection (15 mg/ $/ \mathrm{kg}$, s.c.) one week after chronic morphine treatment significantly increased total dendritic spines density in the NAc shell and core in WT mice, when compared with mice chronically receiving saline (SALINE/SALINE) (Fig. 2; $\left.F_{(1,98)}=4.718 ; p<0.05\right)$ or morphine (MORPHINE/SALINE) (Fig. $\left.2 ; F_{(1,94)}=5.579 ; p<0.05\right)$. In these groups receiving the morphine challenge, the increase in spine density in WT mice was significantly higher in the NAc core when compared to CB1-R KO mice (Fig. 2; $F_{(1,98)}=7.178$; $p<0.01)$. The structural changes observed in these mice were mainly due to an increased density in stubby- $\left(F_{(1,98)}=9.653 ; p<0.01\right)$ and branched-types $\left(F_{(1,98)}=7.955 ; p<0.01\right)$ of spines in the NAc shell (Fig. 4); while in the NAc core these changes relied on an increase in thin-spine density (Fig. 3, Fig. 4 and Table $\left.1 \mathrm{~F}_{(1,94)}=5.006 ; p<0.05\right)$. In contrast, this morphine challenge did not modify total spine density in CB1-R KO mice in both sub-regions of the NAc (Fig. 2), although enhanced the proportion of mushroom-type of spines in the NAc shell (Fig. 3; $\left.F_{(1,89)}=5.196 ; p<0.05\right)$ and mushroom- and stubby-type of spines in the NAc core (Fig. 4; $F_{(1,97)}=6.013 ; p<0.05 ; F_{(1,97)}=5.335 ; p<0.05$, respectively) when compared with control CB1-R KO animals (SALINE/SALINE). In the mPFC, this morphine challenge decreases the prior enhancement of total spine density produced by chronic morphine in both genotypes (Fig. 2, WT $F_{(1,102)}=6.285 ; p<0.05 ; C B 1-R$ KO $\left.F_{(1,87)}=5.176 ; p<0.05\right)$. Therefore, the structural changes in the MPFC produced by chronic morphine treatment and re-exposure do not seem to be dependent on CB1-R. 


\section{DISCUSSION}

This study reveals that morphine-induced behavioral sensitization is associated to specific structural plasticity changes in different areas of the mesocorticolimbic system, a neuronal circuit that plays a crucial role in drug addiction. Several of these modifications induced by morphine were absent or modified in CB1-R KO mice, an animal model in which opioid behavioral sensitization is impaired (Martin et al., 2000; present study). These specific changes in structural plasticity observed in WT, but not in CB1-R KO mice, might play an important role in the maladaptive behavioral alterations that characterize drug addiction.

In agreement with previous studies (Zimmer et al., 1999; Martin et al., 2000), control mice lacking CB1-R showed basal hypolocomotion when compared with WT animals (Fig. 1) and modifications in structural plasticity in the NAc core and shell (Fig. 2) that could be related to this hypolocomotor phenotype. Indeed, previous studies have revealed that alterations in dopaminergic and glutamatergic neurotransmission into the NAc modify locomotion in rodents (Fishman et al., 1983; Pulvirenti et al., 1989). Interestingly, dopamine and glutamate inputs are crucial modulators of NAc MSN activities by making synaptic connections at distal parts of terminal dendrites (Smith and Bolam, 1990; Spooren et al., 1991), which are altered in CB1-R KO mice. These modifications in basal distal dendritic spine density and/or in spine morphology could modify glutamatergic and dopaminergic neurotransmission in both subregions of the NAC, and alter locomotion in CB1-R KO mice. CB1-R also exert direct control of glutamate (Robbe et al., 2001) and dopamine (Hungun et al., 2003) release at the synaptic level in the NAc. The absence of endocannabinoid-modulatory actions on dopamine and/or glutamate release in the NAc can account for the basal locomotor impairments in CB1-R KO mice.

Previous studies have also described similar modifications in basal neuronal plasticity in CB1-R KO mice in other neuronal populations, such as the pyramidal neurons of the motor cortex 
(Ballesteros-Yañez et al., 2007). These results suggest that CB1-R are important mediators of normal neurodevelopmental processes in specific brain areas, in agreement with the role of these receptors in neural progenitor proliferation, axonal navigation and synapse formation during embryonic neuronal development (Fernández-Ruiz et al., 2000; Diaz-Alonso et al., 2012). CB1-R also control dendritic spine dynamics in specific areas of the adult central nervous system, such as the hippocampus, where CB1-R blockade leads to a decrease in total density of spines (Busquets-Garcia et al., 2013). No changes in basal structural plasticity were observed in the mPFC of CB1-R KO mice (Fig. 2 and Fig. 5) suggesting that the role of CB1-R in neurodevelopment seems to be particularly relevant in specific brain areas, such as the NAc, motor cortex or hippocampus.

The hyperlocomotor effects of acute morphine were proportionally 5 times higher in CB1-R KO mice than in WT animals (Fig. 1), in agreement with previous studies demonstrating an involvement of these receptors in opioid-induced pharmacological responses (Ledent et al., 1999, Martin et al., 2000). Several studies have revealed a functional interaction and/or crosstalk between the endogenous cannabinoid and opioid systems regulating different physiological responses, such as nociception, drug reinforcement and anxiety (Maldonado et al., 2006). Dopamine and glutamate neurotransmission into the NAc are important mechanisms involved in acute opioid-induced hyperlocomotion (Steketee and Kalivas, 2011). Deregulations in dopamine and/or glutamate functionality in the NAC of CB1-R KO mice might have affected acute opioid-induced behavioral responses in these deficient animals. Interestingly, a single exposure to morphine modified neuroplasticity in the NAc, but not in the mPFC, in both WT and CB1-R KO mice in a short period of time (30 min). Therefore, these structural plasticity modifications produced by acute morphine seem to occur only in specific areas of the brain reward circuit.

Behavioral- and pharmacological-dependent structural plasticity changes that include modifications in the number or morphology of pre-existing spines are highly dynamic 
processes occurring in short periods of time in different brain areas, such as the NAc (Lendvai et al., 2000; Gipson et al., 2013). These acute plasticity changes were more important in CB1-R KO (increase in total dendritic spine densities in the NAc shell and core) than in WT animals (increase in stubby- and mushroom-type density of spines in the NAc shell and core, respectively). These data suggest that CB1-R participate in neuronal rearrangements promoted in the mesocorticolimbic system by acute morphine administration, and the lack of CB1-R exacerbates these structural plasticity changes.

Alterations in neuronal plasticity after acute drug administration may affect neuronal rearrangements produced by subsequent exposures to the drug that may be crucial for the development of the addictive process. Thus, aberrant dendritic plasticity in CB1-R KO mice induced by acute morphine may also affect neuroplastic modifications after repeated morphine treatment and disrupt the development of behavioral sensitization. In agreement, morphine already induced locomotor sensitization after 3 days of chronic treatment in WT mice, whereas CB1-R KO mice developed locomotor tolerance from the same day of chronic morphine treatment (data not shown).

No major changes in total dendritic spine density or density of specific spine sub-types in the NAc shell or core were observed in WT and CB1-R KO mice one week after the disruption of chronic morphine treatment, although increased proportion of stubby-types spines was revealed in the NAc core in CB1-R KO animals (Fig. 2, Table 2). These results reflect that disruption of chronic morphine treatment might be associated with a progressive waning of structural plasticity established in the NAc during morphine treatment. Similar results have also been described in dendrites of MSN of the NAc after prolonged withdrawal from a chronic cocaine treatment (Dumitriu et al., 2012). In contrast, disruption of chronic morphine treatment was associated with an increase in dendritic spine density, mainly thin spines, in the mPFC in both WT and CB1-R KO mice. This increase was significantly less important in CB1-R KO than in WT mice (Fig. 2 and 3, Table 2). The mPFC glutamatergic projections to the NAc are 
crucial for the expression of drug-induced behavioral sensitization (Steketee and Kalivas, 2011). Thus, we hypothesize that the cessation of chronic morphine treatment induces alterations in structural plasticity in the MPFC that could be crucial for the expression of behavioral sensitization. In the mPFC, a return to the baseline levels in the total spine density was observed in WT and CB1-R KO animals after a challenge morphine injection. This effect was mainly due to a reduction in the number of thin spines in both genotypes, and suggests that neuroplasticity induced in this brain area is a highly dynamic and short lasting process. In agreement with these plasticity changes, morphine re-exposure produced hyperlocomotion in both genotypes, although the effects were attenuated in CB1-R KO animals (Fig. 1). The decrease in the magnitude of the changes in neuroplasticity in the MPFC combined with the absence of regulatory actions of the endocannabinoid system at the level of the NAc synapses in CB1-R KO mice (Robbe et al., 2001; Hungun et al., 2003; Sperlagh et al., 2009) might explain this attenuated response. Morphine challenge after the disruption of the chronic treatment increased the total dendritic spine densities in both NAc core and shell in WT, but not in CB1-R KO animals (Fig. 2). These neuronal rearrangements in WT mice were mainly due to an increase in stubby and branched type spines density in the NAc shell and an increase of thin spines in the NAc core. After this morphine challenge, CB1-R KO only showed an increase in mushroom-type spines in both sub-regions of the NAc and an increase in stubby-spines in the NAc core (Table 1). The increase in branched spines in the shell of the NAc in WT mice is of particular interest since this type of spines is considered an effective mechanism to enhance synaptic efficacy (Rusakov et al., 1996) and may provide an efficient way to increase the input efficacy into the NAc shell and to facilitate opioid-behavioural sensitization.

Previous studies on chronic morphine-induced structural plasticity have reported conflicting results. Most studies have shown a decrease in spine densities in most brain areas after morphine chronic treatment (see Robinson and Kolb, 2004 for review). However, other studies have reported the opposite results, describing an increase in dendritic spine densities in the 
cortex and NAc of mice after chronic morphine exposure (Pal and Das, 2013). Differences in sex, species, experimental procedure, doses regime and the methodology used to evaluate structural plasticity might explain these discrepancies after chronic opioid treatment.

We can summarize that morphine-induced structural plasticity in the NAc and MPFC is a highly dynamic phenomenon that evolves during the time course of the sensitization process. Indeed, a single morphine administration already triggers spines morphology changes in the NAc core and shell, which can potentially participate in the development of behavioral sensitization. Structural plasticity changes were no longer observed in the NAc core after the disruption of chronic morphine treatment, whereas increased spines density was revealed in the MPFC at this time point mainly in WT mice, which could participate in the long-term maintenance of morphine sensitization. Finally, re-exposure to morphine one week after the cessation of the chronic treatment produced additional spine density changes in the NAc in WT, but not in CB1$\mathrm{R}$ KO mice, which parallels the development of morphine behavioral sensitization in these mice. The enhancement of morphine induced structural plasticity after chronic morphine treatment could represent a neuronal mechanism underlying behavioral sensitization. Finally, our results highlight the involvement of CB1-R in the structural plasticity changes induced by morphine in the NAc core and shell and in lesser extent in the mPFC, which can possibly account for the impaired morphine-induced locomotor sensitization observed in CB1-R KO mice. 


\section{ACKNOWLEDGEMENTS}

This work was supported by European Commission-FP7 (\#HEALTH-F2-2013-602891), RETICSInstituto de Salud Carlos III (\#RD12/0028/0023), MICINN-Ministerio de Ciencia e Innovación (\#SAF2011-29864), AGAUR-Generalitat de Catalunya (\#2009SGR00731 and \#2014-SGR-1547). FEDER co-funding is also acknowledged. 


\section{AUTHORS CONTRIBUTIONS}

TG and JPC conducted all experiments. TG, MM and RM participated in experimental design and wrote the manuscript. MM, TG and RM participated in the interpretation of the data. 


\section{REFERENCES}

Ballesteros-Yáñez I, Valverde O, Ledent C, Maldonado R, DeFelipe J (2007) Chronic cocaine treatment alters dendritic arborization in the adult motor cortex through a CB1 cannabinoid receptor-dependent mechanism. Neuroscience 146:1536-1545.

Busquets-Garcia A, Gomis-González M, Guegan T, Agustín-Pavón C, Pastor A, Mato S, PérezSamartín A, Matute C, de la Torre R, Dierssen M, Maldonado R, Ozaita A (2013) Targeting the endocannabinoid system in the treatment of fragile X syndrome. Nat Med 19:603-607.

Buxbaum AR, Wu B, Singer RH (2014) Single $\beta$-actin mRNA detection in neurons reveals a mechanism for regulating its translatability. Science 343:419-422.

Crombag HS, Gorny G, Li Y, Kolb B, Robinson TE (2005) Opposite effects of amphetamine selfadministration experience on dendritic spines in the medial and orbital prefrontal cortex. Cereb Cortex 15:341-348.

De Petrocellis L, Di Marzo V (2009) An introduction to the endocannabinoid system: from the early to the latest concepts. Best Pract Res Clin Endocrinol Metab 23:1-15.

Díaz-Alonso J, Guzmán M, Galve-Roperh I (2012) Endocannabinoids via CB $_{1}$ receptors act as neurogenic niche cues during cortical development. Philos Trans $R$ Soc Lond B Biol Sci 367:3229-3241.

Dumitriu D, Laplant Q, Grossman YS, Dias C, Janssen WG, Russo SJ, Morrison JH, Nestler EJ (2012) Subregional, dendritic compartment, and spine subtype specificity in cocaine regulation of dendritic spines in the nucleus accumbens. J Neurosci 32:6957-6966.

Enriquez-Barreto L, Cuesto G, Dominguez-Iturza N, Gavilán E, Ruano D, Sandi C, Fernández-Ruiz A, Martín-Vázquez G, Herreras O, Morales M (2014) Learning improvement after PI3K 
activation correlates with de novo formation of functional small spines. Front Mol Neurosci $6: 54$

Fernández-Ruiz J, Berrendero F, Hernández ML, Ramos JA (2000) The endogenous cannabinoid system and brain development. Trends Neurosci 23:14-20.

Fishman RH, Feigenbaum JJ, Yanai J, Klawans HL (1983) The relative importance of dopamine and norepinephrine in mediating locomotor activity. Prog Neurobiol 20:55-88.

Gipson CD, Reissner KJ, Kupchik YM, Smith AC, Stankeviciute N, Hensley-Simon ME, Kalivas PW (2013) Reinstatement of nicotine seeking is mediated by glutamatergic plasticity. Proc Natl Acad Sci U S A 110:9124-9129.

Grutzendel J, Tsai J, Gan WB (2003) Rapid labeling of neuronal populations by ballistic delivery of fluorescent dyes. Methods 30:79-85.

Guegan T, Cutando L, Ayuso E, Santini E, Fisone G, Bosch F, Martinez A, Valjent E, Maldonado R, Martin (2013) Operant behavior to obtain palatable food modifies neuronal plasticity in the brain reward circuit. Eur Neuropsychopharmacol 23:146-159.

Harris KM, Kater SB (1994) Dendritic spines: cellular specializations imparting both stability and flexibility to synaptic function. Annu Rev Neurosci 17:341-371.

Hungund BL, Szakall I, Adam A, Basavarajappa BS, Vadasz C (2003) Cannabinoid CB1 receptor knockout mice exhibit markedly reduced voluntary alcohol consumption and lack alcoholinduced dopamine release in the nucleus accumbens. J Neurochem 84:698-704.

Hyman SE, Malenka RC (2001) Addiction and the brain: the neurobiology of compulsion and its persistence. Nat Rev Neurosci 2:695-703. 
Kalivas PW, O'Brien C (2008) Drug addiction as a pathology of staged neuroplasticity. Neuropsychopharmacology 33:166-80.

Koob GF, Le Moal M (2001) Drug addiction, dysregulation of reward, and allostasis. Neuropsychopharmacology 24:97-129.

Ledent C, Valverde O, Cossu G, Petitet F, Aubert JF, Beslot F, Böhme GA, Imperato A, Pedrazzini T, Roques BP, Vassart G, Fratta W, Parmentier M (1999) Unresponsiveness to cannabinoids and reduced addictive effects of opiates in CB1 receptor knockout mice. Science 283:401-404.

Lee KW, Kim Y, Kim AM, Helmin K, Nairn AC, Greengard P (2006) Cocaine-induced dendritic spine formation in D1 and D2 dopamine receptor-containing medium spiny neurons in nucleus accumbens. Proc Natl Acad Sci U S A 103:3399-3404.

Lendvai B, Stern EA, Chen B, Svoboda K (2000) Experience-dependent plasticity of dendritic spines in the developing rat barrel cortex in vivo. Nature 404:876-881.

Leuner B, Falduto J, Shors TJ (2003) Associative memory formation increases the observation of dendritic spines in the hippocampus. J Neurosci 23:659-665.

Li Y, Acerbo MJ, Robinson TE (2004) The induction of behavioural sensitization is associated with cocaine-induced structural plasticity in the core (but not shell) of the nucleus accumbens. Eur J Neurosci 20:1647-1654.

Lüscher C, Malenka RC (2011) Drug-evoked synaptic plasticity in addiction: from molecular changes to circuit remodeling. Neuron 69:650-663.

Maldonado R, Valverde O, Berrendero F (2006) Involvement of the endocannabinoid system in drug addiction. Trends Neurosci 29:225-232. 
Martin M, Ledent C, Parmentier M, Maldonado R, Valverde O (2000) Cocaine, but not morphine, induces conditioned place preference and sensitization to locomotor responses in CB1 knockout mice. Eur J Neurosci 12:4038-4046.

Pal A, Das S (2013) Chronic morphine exposure and its abstinence alters dendritic spine morphology and upregulates Shank1. Neurochem Int 62:956-964.

Paxinos and Franklin (2001) The mouse brain in stereotaxic coordinates. San Diego: Academic Press.

Pulvirenti L, Swerdlow NR, Koob GF (1989) Microinjection of a glutamate antagonist into the nucleus accumbens reduces psychostimulant locomotion in rats. Neurosci Lett 103:213-218.

Rampon C, Tsien JZ (2000) Genetic analysis of learning behavior-induced structural plasticity. Hippocampus 10:605-609.

Robbe D, Alonso G, Duchamp F, Bockaert J, Manzoni OJ (2001) Localization and mechanisms of action of cannabinoid receptors at the glutamatergic synapses of the mouse nucleus accumbens. J Neurosci 21:109-116.

Robinson TE, Berridge KC (2003) Addiction. Annu Rev Psychol 54:25-53.

Robinson TE, Kolb B (1997) Persistent structural modifications in nucleus accumbens and prefrontal cortex neurons produced by previous experience with amphetamine. J Neurosci 17(21):8491-8497.

Robinson TE, Kolb B (2004) Structural plasticity associated with exposure to drugs of abuse. Neuropharmacology 47 S1:33-46. 
Rusakov DA, Stewart MG, Korogod SM (1996) Branching of active dendritic spines as a mechanism for controlling synaptic efficacy. Neuroscience 75:315-323.

Russo SJ, Dietz DM, Dumitriu D, Morrison JH, Malenka RC, Nestler EJ (2010) The addicted synapse: mechanisms of synaptic and structural plasticity in nucleus accumbens. Trends Neurosci 33:267-276.

Smith AD, Bolam JP (1990) The neural network of the basal ganglia as revealed by the study of synaptic connections of identified neurones. Trends Neurosci 13:259-265.

Sperlágh B, Windisch K, Andó RD, Sylvester Vizi E (2009) Neurochemical evidence that stimulation of CB1 cannabinoid receptors on GABAergic nerve terminals activates the dopaminergic reward system by increasing dopamine release in the rat nucleus accumbens. Neurochem Int 54:452-457.

Spooren WP, Veening JG, Groenewegen HJ, Cools AR (1991) Efferent connections of the striatopallidal and amygdaloid components of the substantia innominata in the cat: projections to the nucleus accumbens and caudate nucleus. Neuroscience 44:431-447.

Steketee JD, Kalivas PW (2011) Drug wanting: behavioral sensitization and relapse to drugseeking behavior. Pharmacol Rev 63:348-365.

Vanderschuren LJ, Kalivas PW (2000) Alterations in dopaminergic and glutamatergic transmission in the induction and expression of behavioral sensitization: a critical review of preclinical studies. Psychopharmacology (Berl) 151:99-120.

Vanderschuren LJ, Pierce RC (2010) Sensitization processes in drug addiction. Curr Top Behav Neurosci 3:179-195. 
Zimmer A, Zimmer AM, Hohmann AG, Herkenham M, Bonner TI (1999) Increased mortality, hypoactivity, and hypoalgesia in cannabinoid CB1 receptor knockout mice. Proc Natl Acad Sci U S A 96:5780-5785. 


\section{FIGURE LEGENDS}

FIGURE 1: Genetic deletion of CB1-R alters basal locomotor activity, acute morphine hyperlocomotor effects and the development and expression of locomotor sensitization to morphine. (A) Basal locomotor activity of WT (white columns; $n=60$ ) and CB1-R KO (black columns; $n=59$ ) mice during the three days of habituation. (B) Hyperlocomotor effects of morphine (15 mg/kg, s.c.) in WT and CB1-R KO mice. Day 4 represents morphine acute effects morphine, day 19 represents the behavioral sensitization at the end of the chronic treatment (development phase), and the maintenance or late expression of morphine sensitization is shown on day 26 . From day 4 to 19 , mice of each genotype were treated, twice daily, with morphine (black columns; WT, $n=31$; CB1-R KO, $n=28$ ) or saline (white columns; WT, $n=29$; CB1-R KO, n=31). At day 26, after 1 week without treatment, mice chronically treated with saline received either an injection of saline (white columns; WT, $n=16 ; C B 1-R K O, n=17$ ), or morphine (upright stripped columns; WT, $n=13 ; C B 1-R$ KO, $n=14$ ). Similarly, mice chronically treated with morphine received, at day 26, either an injection of saline (downright stripped columns; WT, n=12; CB1-R KO, n=12) or morphine (black columns; WT, $n=19 ; C B 1-R K O, n=16$ ). (C) Table showing the percentage of increase or decrease of locomotor activity in WT and CB1$\mathrm{R}$ KO mice, triggered by the habituation procedure (HAB: comparison of locomotor activity at day 3 vs. day 1), acute morphine injection (ACUTE: comparison of the locomotor activity of morphine-treated animal at day 4 vs. their basal activity at day 3), last injection of the morphine chronic treatment (DEVELOPMENT: comparison of morphine hyperlocomotor effects at day 19 vs. day 4), and challenge morphine injection one week after cessation of the chronic treatment (MAINTENANCE: comparison of morphine hyperlocomotor effects at day 26 vs. day 4). Data are expressed as mean of the total number of photocells beam crossed during the 15 min session \pm s.e.m. $\star \mathrm{P}<0.05, \star \star \mathrm{P}<0.01, \star \star \star \mathrm{P}<0.001$ (WT vs. CB1-R KO, Fisher F-

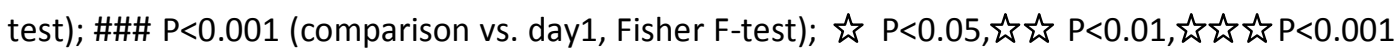


(morphine-treated vs. saline-treated mice, Fisher F-test); @ P<0.05, @@P<0.01, @@@ $\mathrm{P}<0.001$ (comparison vs. day 4, Fisher F-test).

FIGURE 2: Structural plasticity changes induced by morphine in the mesocorticolimbic circuit are dependent on CB1-R. Quantification of total dendritic spines density (number/10 $\mu m$ ) in MSN from the NAc shell $(\mathbf{A})$ and core $(\mathbf{C})$ and basilar dendrites in pyramidal neurons of the mPFC (E) in WT and CB1-R KO mice chronically treated with saline and receiving at day 26 an injection of saline (white columns, Chronic Sal/Acute Sal) or morphine (upright stripped columns; Chronic Sal/Acute Mor); and of WT and CB1-R KO mice chronically treated with morphine and receiving at day 26 an injection of saline (downright stripped columns; Chronic Mor/Acute Sal) or morphine (black columns, Chronic Mor/Acute Mor and Mor/Mor). Data represent the average of 42-52 dendrites per experimental group; a total of 6-8 dendrites per animal ( $n=7-8$ per experimental group) and brain area were analysed, and no more than 2 dendrites were evaluated from the same neuron. Illustrations of Dil-labeled dendrites of MSN in the NAc shell (B), NAc core (D) and Dil-labeled basilar dendrites of pyramidal neurons in the mPFC (F) of the different experimental groups of WT and CB1-R KO mice (Sal/Sal represents mice exposed to Chronic Sal/Acute Sal; Sal/Mor represents mice exposed to Chronic Sal/Acute Mor; Mor/Sal represents mice exposed to Chronic Mor/Acute Sal and Mor/Mor represents mice exposed to Chronic Mor/Acute Mor). Data are expressed as mean \pm s.e.m. $\star \mathrm{P}<0.05, \star \star \mathrm{A}<0.01$ (WT vs. CB1-R KO, Fisher F-test); \# P<0.05, (WT vs. CB1-R KO, Fisher Ftest); \$ $\mathrm{P}<0.05$ (comparison vs. Sal/Sal, Fisher F-test); 约 $\mathrm{P}<0.05$, 我㧒 $\mathrm{P}<0.01$ (comparison vs. Sal/Sal, Fisher F-test); \& P<0.05 (comparison vs. Mor/Sal, Fisher F-test); @P<0.05 (comparison vs. Mor/Sal, Fisher F-test).

FIGURE 3: Spines subtypes analysis in the NAc shell in the different experimental groups of WT and CB1-R KO mice. Quantification of the density (number/10 $\mu \mathrm{m}$ ) of thin, stubby, mushroom, wide and branched spines in MSN of the NAc shell in the different experimental 
groups of WT (A) and CB1-R KO mice (B). Data represent the average of 42-52 dendrites per experimental group; a total of 6-8 dendrites per animal ( $n=7-8$ per experimental group) were analysed, and no more than 2 dendrites were evaluated from the same neuron. Data are expressed as mean \pm s.e.m. $\star \mathrm{P}<0.05$ (WT vs CB1-R KO, Fisher F-test); 论话 $\mathrm{P}<0.01$ (comparison vs. Sal/Sal, Fisher F-test); $\$ \mathrm{P}<0.05, \$ \$ \mathrm{P}<0.01$ (comparison vs. Sal/Sal, Fisher F-test).

FIGURE 4: Spines subtypes analysis in the NAc core in the different experimental groups of WT and CB1-R KO mice. Quantification of the density (number/10 $\mu \mathrm{m}$ ) of thin, stubby, mushroom, wide and branched spines in MSN of the NAc core in the different experimental groups of WT (A) and CB1-R KO mice (B). Data represents the average of 42-52 dendrites per experimental group; a total of 6-8 dendrites per animal ( $n=7-8$ per experimental group) were analysed, and no more than 2 dendrites were evaluated from the same neuron. Data are

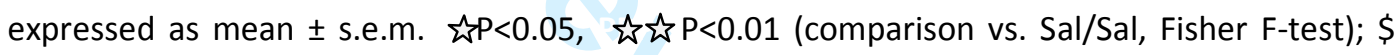
$\mathrm{P}<0.05$ (comparison vs. Sal/Sal, Fisher F-test); \&\& $\mathrm{P}<0.01$ (comparison vs. Mor/Sal, Fisher Ftest).

FIGURE 5: Spines subtypes analysis in the MPFC in the different experimental groups of WT and CB1-R KO mice. Quantification of the density (number/10 $\mu \mathrm{m}$ ) of thin, stubby, mushroom, wide and branched spines in pyramidal neurons of the MPFC in the different experimental groups of WT (A) and CB1-R KO mice (B). Data represents the average of 42-52 dendrites per experimental group; a total of 6-8 dendrites per animal ( $n=7-8$ per experimental group) were analysed; and no more than 2 dendrites were evaluated from the same neuron. Data are

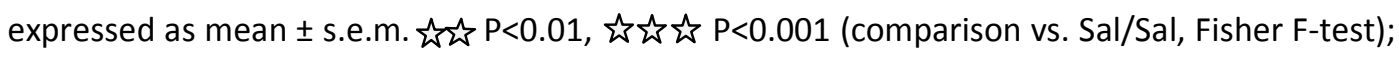
\& $\mathrm{P}<0.05$ (comparison vs. Mor/Sal, Fisher F-test).

TABLE 1: Statistical analysis of habituation and locomotor effects of morphine in WT and CB1-R KO mice. 


\section{TABLE 2: Statistical analysis of the effects of morphine on dendritic spines density in the NAC} shell, core and MPFC in WT and CB1-R KO mice.

TABLE 3: Summary of main results. Acute morphine treatment increases to a greater extent locomotor activity in CB1-R KO mice (++) than WT mice (+) (in comparison to their respective basal activity at day 3). This treatment induces spine morphology changes in the NAc shell and core of WT mice (higher proportion of stubby and mushroom-types spines in the NAc shell and core, respectively), while this acute injection leads to enhance total spine density in those two brain areas in CB1-R KO mice (through mainly an increment of mushroom and stubby-types in the NAc shell and core, respectively). During the chronic morphine treatment, the hyperlocomotor effects of morphine are further enhanced in WT mice, while these effects significantly diminish in CB1-R KO mice (in comparison to the acute effects at day 4). One week after cessation of the morphine chronic treatment, WT and CB1-R KO mice present both an increase of total spine density in the MPFC (through mainly an increment of thin-types spines, and also stubby ones for CB1-R KO mice). At this same time point, re-exposure to morphine leads to a greater enhancement of locomotor activity in WT mice than after the acute injection at day 4 or during the chronic treatment (day 7-10-13-16-19)). Similarly, this challenge injection of morphine produces higher hyperlocomotor effects than an acute injection (day 4) in CB1-R KO mice, although to a smaller extent than WT mice. Re-exposure to morphine reduces to a basal level the total spine density increment observed in the MPFC in WT and CB1-R KO mice one week after cessation of the chronic morphine treatment. Moreover, this challenge injection increases the total spine density in the NAc shell and core in WT mice, but not in CB1-R KO mice in which only spine morphology changes are observed in those brain areas (higher proportion of mushroom and stubby-types spines in the NAc shell and core, respectively). 
FIGURE 1
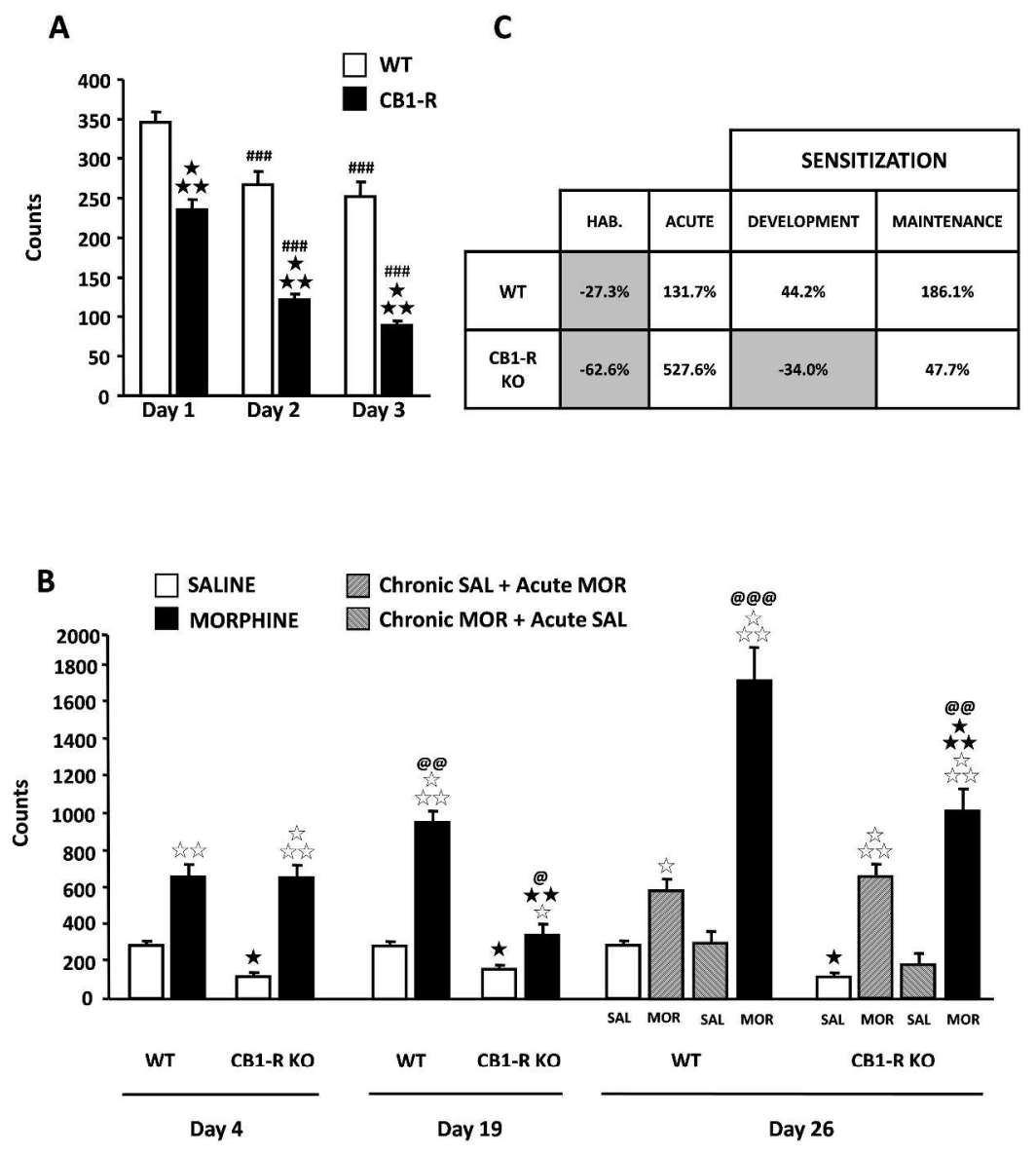

Genetic deletion of CB1-R alters basal locomotor activity, acute morphine hyperlocomotor effects and the development and expression of locomotor sensitization to morphine. $1190 \times 1719 \mathrm{~mm}(96 \times 96 \mathrm{DPI})$ 
FIGURE 2
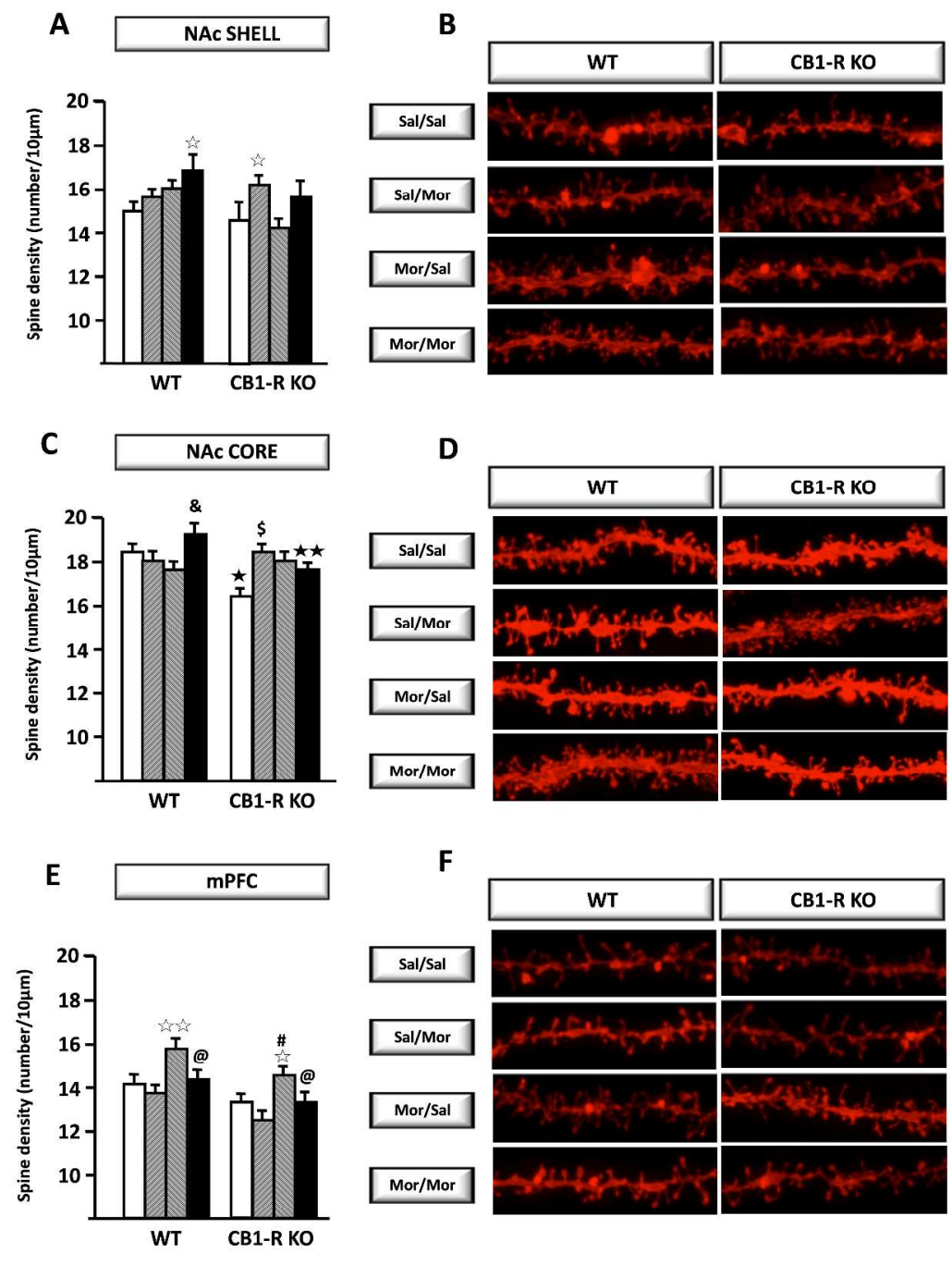

$\square$ chronic SAL/Acute SAL $\square$ Chronic SAL/Acute MOR $\square$ Chronic MOR/ACute SAL

Chronic MOR/Acute MOR

Structural plasticity changes induced by morphine in the mesocorticolimbic circuit are dependent on CB1-R. $1190 \times 1719 \mathrm{~mm}(96 \times 96 \mathrm{DPI})$ 


\section{FIGURE 3}
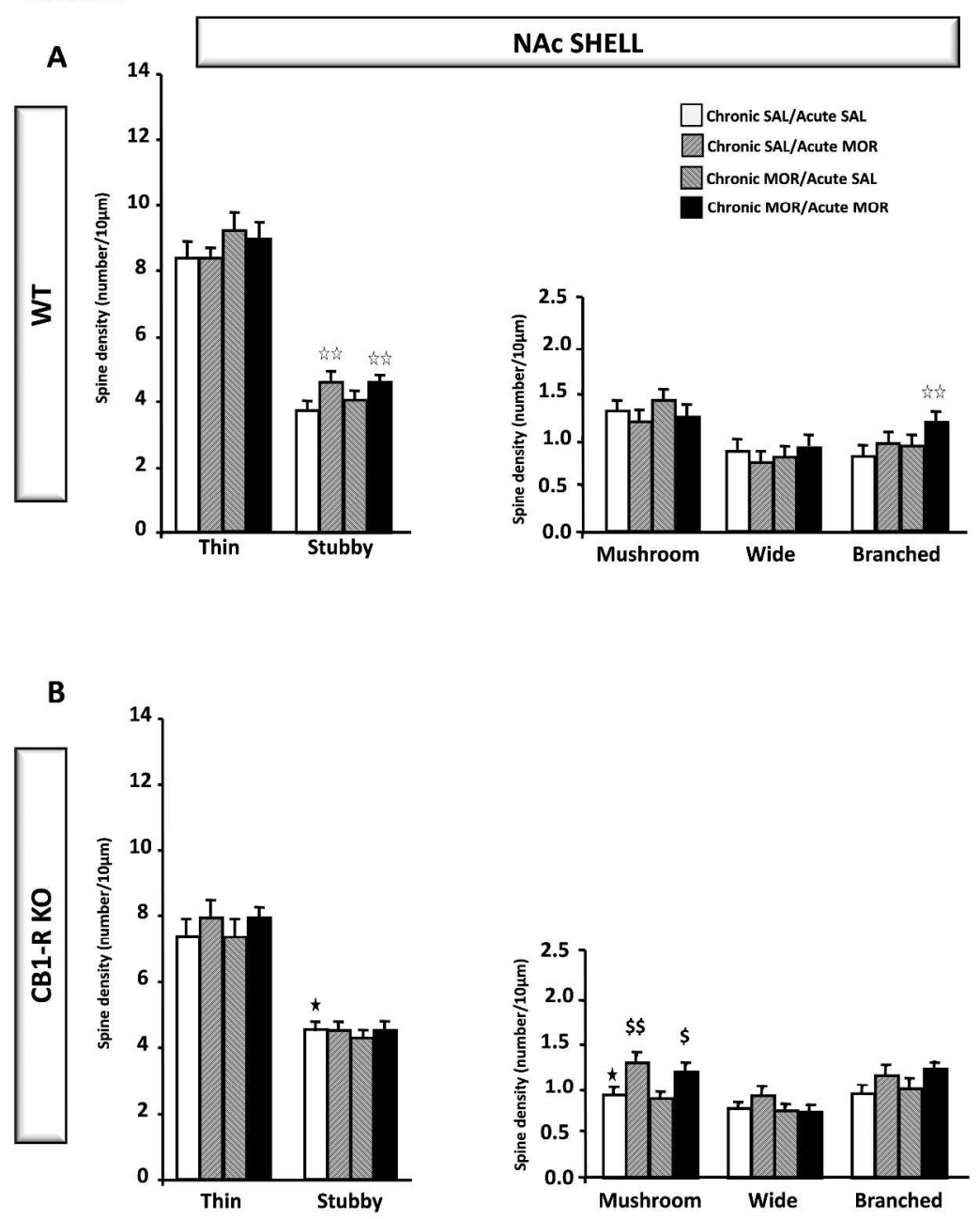

Spines subtypes analysis in the NAc shell in the different experimental groups of WT and CB1-R KO mice. $1190 \times 1719 \mathrm{~mm}(96 \times 96 \mathrm{DPI})$ 
FIGURE 4
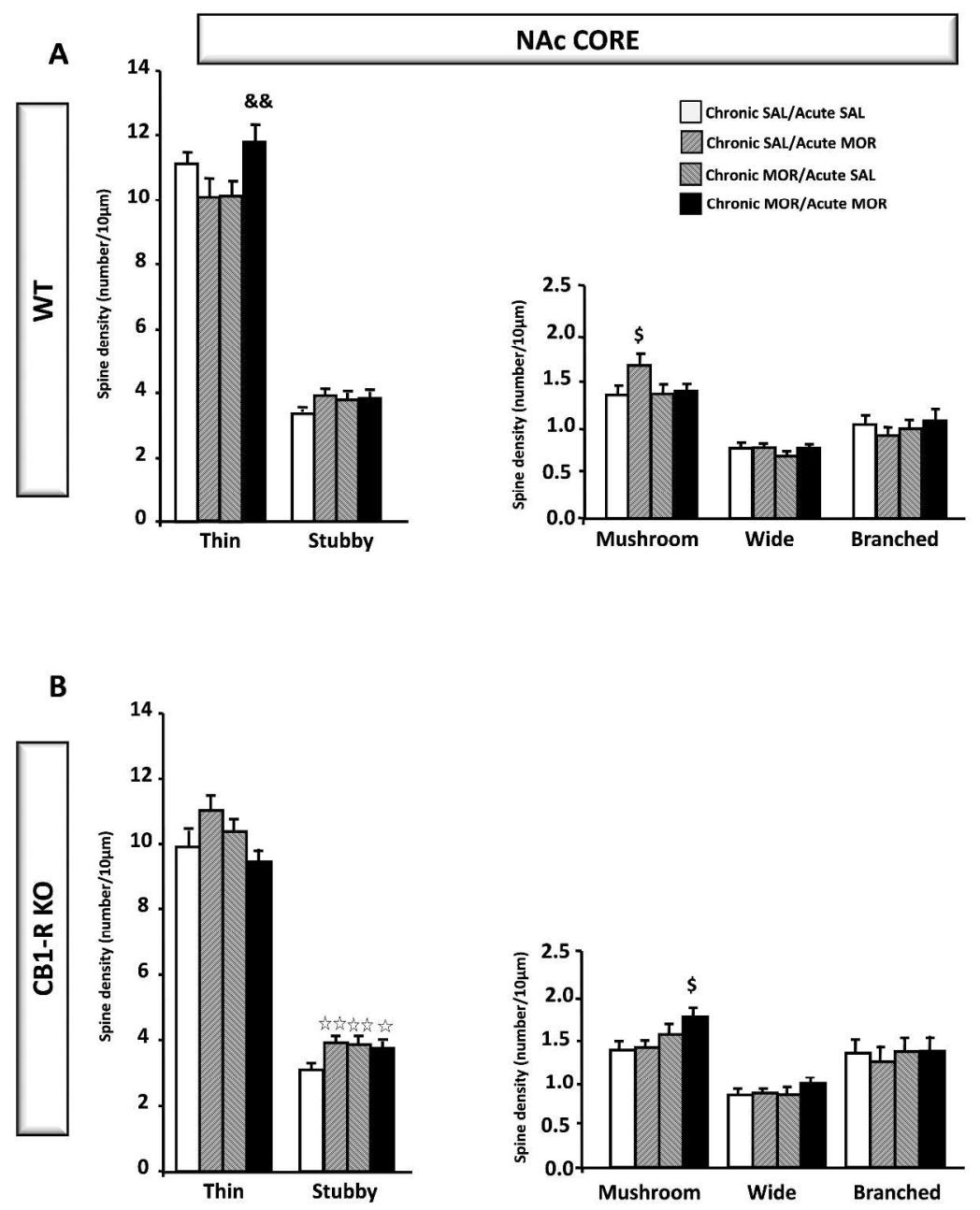

Spines subtypes analysis in the NAc core in the different experimental groups of WT and CB1-R KO mice. $1190 \times 1719 \mathrm{~mm}(96 \times 96 \mathrm{DPI})$ 


\section{FIGURE 5}
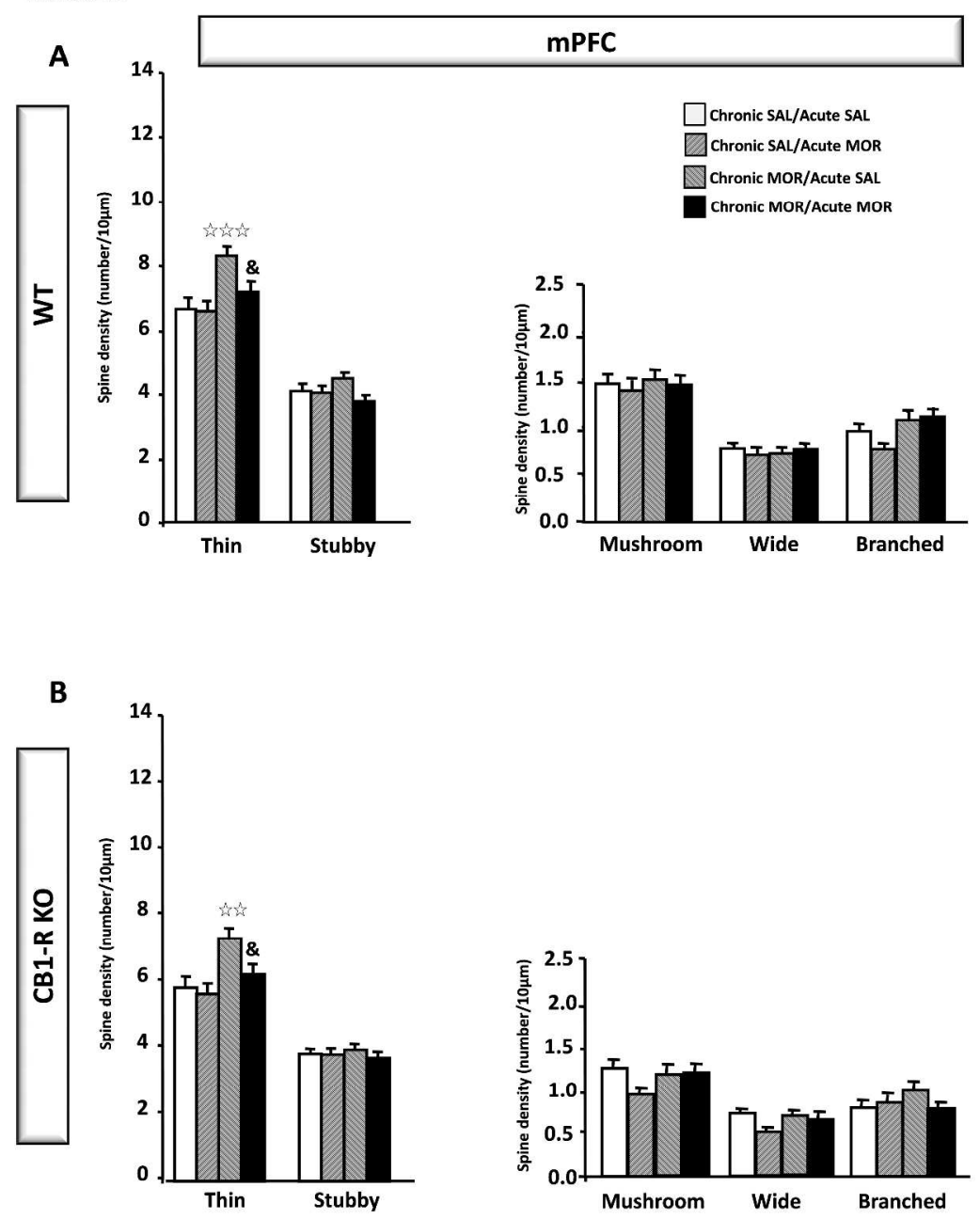

Spines subtypes analysis in the MPFC in the different experimental groups of WT and CB1-R KO mice $1190 \times 1719 \mathrm{~mm}(96 \times 96 \mathrm{DPI})$ 
TABLE 1

\begin{tabular}{|c|c|c|c|c|c|c|}
\hline & \multirow{2}{*}{\multicolumn{2}{|c|}{$\begin{array}{l}\text { Two-way ANOVA } \\
\text { Habituation (Figure 26A) }\end{array}$}} & \multicolumn{4}{|l|}{ Three-way ANOVA } \\
\hline & & & \multicolumn{2}{|c|}{ Sensitization (Figure 26B) } & \multicolumn{2}{|c|}{ Chronic treament (Figure 27A and B) } \\
\hline & Total movements & P-value & Total movements & P-value & Total movements & P-value \\
\hline Genotype (G) & $F(1,94)=79.5318$ & $P<0.001$ & $F(1,111)=40.3113$ & $P<0.001$ & $F(1,115)=59.8966$ & $P<0.001$ \\
\hline Day (D) & $F(2,232)=107.0572$ & $P<0.001$ & $F(2,222)=34.8736$ & $P<0.001$ & $F(5,575)=3.3810$ & $\mathrm{P}<0.01$ \\
\hline Treatment ( $\mathrm{T}$ ) & - & - & $F(3,111)=67.0171$ & $P<0.001$ & $F(1,115)=112.2351$ & $P<0.001$ \\
\hline$G \times D$ & $F(2,232)=4.8896$ & $P<0.01$ & $F(2,222)=12.6161$ & $P<0.001$ & $F(5,575)=16.6058$ & $P<0.001$ \\
\hline GXT & - & - & $F(3,111)=9.7889$ & $P<0.001$ & $F(1,115)=11.5672$ & $P<0.001$ \\
\hline$T \times D$ & - & - & $F(6,222)=12.6161$ & $P<0.001$ & $F(5,575)=2.2221$ & n.s. \\
\hline GXTXD & - & - & $F(6,222)=9.8936$ & $P<0.001$ & $F(5,575)=13.8514$ & $P<0.001$ \\
\hline
\end{tabular}

Statistical analysis of habituation and locomotor effects of morphine in WT and CB1-R KO mice. $1250 \times 937 \mathrm{~mm}(96 \times 96 \mathrm{DPI})$ 
TABLE 2

\begin{tabular}{|c|c|c|c|c|c|c|}
\hline & \multicolumn{6}{|l|}{ Two-way ANOVA } \\
\hline & \multicolumn{6}{|l|}{ Spine density } \\
\hline & Genotype & P-value & Treatment & P-value & Interaction & P-value \\
\hline \multicolumn{7}{|l|}{ Shell } \\
\hline Total & $F(1,349)=3.628$ & n.s. & $F(3,349)=2.668$ & $P<0.05$ & $F(3,349)=1.678$ & n.s. \\
\hline Thin & $F(1,349)=10.548$ & $P<0.01$ & $F(3,349)=0.948$ & n.s. & $F(3,349)=1.153$ & n.s. \\
\hline Stubby & $F(1,349)=6.986$ & $P<0.01$ & $F(3,349)=2.764$ & $P<0.05$ & $F(3,349)=2.540$ & n.s. \\
\hline Mushroom & $F(1,349)=11.249$ & $P<0.001$ & $F(3,349)=0.773$ & n.s. & $F(3,349)=6.049$ & $\mathrm{P}<0.001$ \\
\hline Wide & $F(1,349)=1.020$ & n.s. & $F(3,349)=0.202$ & n.s. & $F(3,349)=2.942$ & $P<0.05$ \\
\hline Branched & $F(1,349)=1.82898$ & n.s. & $F(3,349)=4.0580$ & $P<0.01$ & $F(3,349)=0.0839$ & n.s. \\
\hline \multicolumn{7}{|l|}{ Core } \\
\hline Total & $F(1,363)=4.588$ & $P<0.05$ & $F(3,363)=\mathbf{1 . 2 0 4}$ & n.s. & $F(3,363)=2.839$ & $P<0.05$ \\
\hline Thin & $\mathbf{F}(1,363)=4.479$ & $P<0.05$ & $F(3,363)=0.276$ & n.s. & $F(3,363)=5.182$ & $P<0.01$ \\
\hline Stubby & $F(1,363)=0.525$ & n.s. & $F(3,363)=4.584$ & $P<0.01$ & $F(3,363)=0.181$ & n.s. \\
\hline Mushroom & $F(1,363)=6.032$ & $P<0.05$ & $F(3,363)=1.620$ & n.s. & $F(3,363)=3.283$ & $P<0.05$ \\
\hline Wide & $F(1,363)=0.063$ & n.s. & $F(3,363)=1.203$ & n.s. & $F(3,363)=0.608$ & n.s. \\
\hline Branched & $F(1,363)=1.5338$ & n.s. & $F(3,363)=0.4647$ & n.s. & $F(3,363)=0.0233$ & n.s. \\
\hline \multicolumn{7}{|l|}{ mPFC } \\
\hline Total & $F(1,365)=13.571$ & $P<0.001$ & $F(3,365)=8.954$ & $P<0.001$ & $F(3,365)=0.062$ & n.s. \\
\hline Thin & $F(1,365)=14.753$ & $P<0.001$ & $F(3,365)=11.162$ & $P<0.001$ & $F(3,365)=0.018$ & n.s. \\
\hline Stubby & $F(1,365)=3.228$ & n.s. & $F(3,365)=2.522$ & n.s. & $F(3,365)=0.549$ & n.s. \\
\hline Mushroom & $F(1,365)=5.125$ & $P<0.05$ & $F(3,365)=1.732$ & n.s. & $F(3,365)=0.739$ & n.s. \\
\hline Wide & $F(1,365)=0.509$ & n.s. & $F(3,365)=2.617$ & n.s. & $F(3,365)=1.209$ & n.s. \\
\hline Branched & $F(1,365)=0.1104$ & n.s. & $F(3,365)=2.3171$ & n.s. & $F(3,365)=1.9605$ & n.s. \\
\hline
\end{tabular}

Statistical analysis of the effects of morphine on dendritic spines density in the NAc shell, core and mPFC in WT and CB1-R KO mice $1250 \times 937 \mathrm{~mm}(96 \times 96 \mathrm{DPI})$ 
TABLE 3

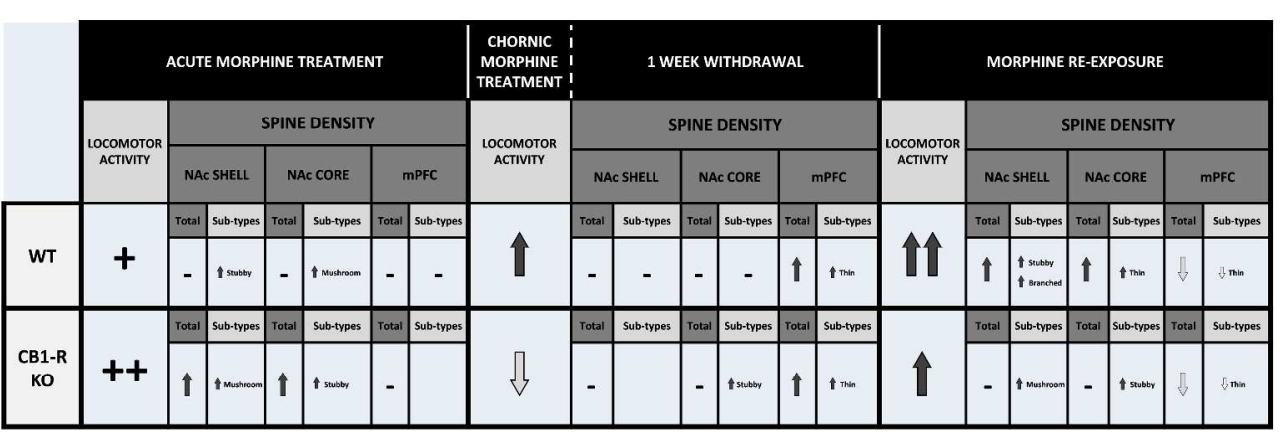

Summary of main results.

$1719 \times 1190 \mathrm{~mm}$ (96 x 96 DPI) 